\title{
Lactobacillus plantarum CAU1055 ameliorates inflammation in lipopolysaccharide-induced RAW264.7 cells and a dextran sulfate sodium-induced colitis animal model
}

\author{
Sun-Hae Choi, ${ }^{1}$ Sun-Hee Lee, ${ }^{1}$ Min Gon Kim, ${ }^{1}$ Hong Jin Lee, ${ }^{2 *}$ and Geun-Bae Kim ${ }^{1 *}$ \\ ${ }^{1}$ Department of Animal Science and Technology, Chung-Ang University, Anseong 17546, Korea \\ ${ }^{2}$ Department of Food Science and Technology, Chung-Ang University, Anseong 17546, Korea
}

\section{ABSTRACT}

This study aimed to screen lactic acid bacteria (LAB) for their anti-inflammatory activity by using RAW264.7 cells and dextran sulfate sodium (DSS)-induced colitis. In all, 192 LAB strains were isolated from healthy human feces, of which 8 strains showed excellent nitric oxide (NO) inhibitory activity. Peptidoglycan extracts of these $8 \mathrm{LAB}$ strains were subjected to NO assay, Western blot, and ELISA. Among the 8 tested strains, extracts of 4 strains significantly inhibited the production of NO, related enzyme activities such as inducible nitric oxide synthase and cyclooxygenase 2, and key cytokines such as tumor necrosis factor- $\alpha$ and IL-6 in RAW264.7 cells. The 4 strains belonged to Lactobacillus (CAU1054, CAU1055, CAU1064, and CAU1301). Oral administration of the 4 strains inhibited DSS-induced body weight loss, colon shortening, and colon damage in ICR mice. The colon tissue of the mice treated with Lactobacillus plantarum strain CAU1055 had significantly reduced levels of inducible nitric oxide synthase, cyclooxygenase 2, tumor necrosis factor- $\alpha$, and IL-6. We found that strain CAU1055 could be used as a candidate probiotic strain for the prevention and treatment of inflammatory bowel disease. Further studies are warranted to confirm the mechanisms of interaction between peptidoglycan of L. plantarum strain CAU1055 and upstream cellular signaling mediators.

Key words: lactic acid bacteria, peptidoglycan, RAW264.7 cells, dextran sulfate sodium, antiinflammatory effect

\section{INTRODUCTION}

Inflammation is a host defense mechanism against tissue injury caused by bacterial infections and chemi-

Received December 21, 2018.

Accepted April 5, 2019.

. $\mathrm{kr}$

*Corresponding authors: hongjin@cau.ac.kr and kimgeun@cau.ac cal or physical damage; it is mainly regulated by proinflammatory mediators such as inducible nitric oxide synthase (iNOS) and cyclooxygenase-2 (COX-2) and enhanced by proinflammatory cytokines including tumor necrosis factor- $\alpha$ (TNF- $\alpha$ ) and IL-6 (Dinarello, 2006; Medzhitov, 2008). Inflammatory bowel diseases (IBD) such as ulcerative colitis and Crohn's disease are chronic inflammatory diseases of the gastrointestinal tract characterized by various symptoms, including rectal bleeding, diarrhea, abdominal pain, fever, and weight loss. Although the etiology of IBD is not yet known, many studies have identified therapeutic molecular targets to treat IBD. For example, Atreya et al. (2000) reported that the soluble IL-6 receptor signaling pathway is involved in inducing resistance to mucosal T-cell apoptosis in patients with Crohn's disease. Similarly, neutrophil transmigration through the apical junction complex was suggested as a potential strategy to regulate mucosal inflammation (Chin and Parkos, 2006). Monoclonal antibodies against TNF- $\alpha$ or integrin and anti-inflammatory drugs such as 5-aminosalicylates and corticosteroids have been approved for the treatment of IBD by the US Food and Drug Administration. However, the development of safe and economical agents without adverse effects is required to prevent IBD.

Probiotics are defined as living microorganisms that provide health-beneficial functions to hosts; they play critical roles in maintaining the intestinal ecosystem, preventing diarrhea, lowering serum cholesterol, and modulating the immune system. Lactic acid bacteria (LAB) are gram-positive, nonsporulating rod- or coccishaped bacteria. Among the LAB, Lactobacillus spp. are widely used in the food and dairy industry and have immunomodulatory functions such as anticancer and anti-inflammatory activities. Among different probiotics, the physiological health-beneficial effects of Lactobacillus and Bifidobacterium have been widely elucidated in diverse chronic diseases, including inflammation. Kim et al. (2015) reported that Lactobacillus helveticus regulated inflammatory T-cell activity and 
suppressed rheumatoid arthritis. Lactobacillus rhamnosus GG was found to modulate the immune responses of dendritic cells, macrophages, and monocytes, and to ameliorate inflammatory diseases (Fong et al., 2015). In clinical trials, Lactobacillus and Bifidobacterium strains were shown to maintain remission and prevent relapse in patients with Crohn's disease (Hijova and Soltesova, 2013).

Several strains of Lactobacillus plantarum have been reported to exhibit some ameliorative effects on inflammation in LPS-induced cell lines and a dextran sulfate sodium (DSS)-induced colitis animal model (Liu et al., 2011; Cui et al., 2016; Wang et al., 2019). However, different $L$. plantarum strains may exert different positive effects on ulcerative colitis in vivo. It is well known that many probiotic properties are strain-specific, meaning that different strains of the same bacterial genus and species may exert diverse probiotic properties. This is why the specific probiotic effects of each individual LAB strains should be well defined, and the effect of each strain should be demonstrated case by case (Campana et al., 2017). Accumulating evidence firmly indicates that probiotics might prevent inflammatory immune malfunctions, including IBD. Thus, significant efforts have been made to screen intestinal microorganisms and identify novel strains that can modulate inflammation.

Although Lactobacillus is known to regulate immunity, the molecular mechanisms are not yet elucidated (Weiss et al., 2010). Microbe-associated molecular patterns (MAMP) such as peptidoglycans (PGN) and teichoic acid of Lactobacillus are associated with the reaction of pattern recognition receptors (PRR) such as toll-like receptors (TLR) in intestinal epithelial cells; these interactions act to regulate immune functions (Ebrahimi and Jafarei, 2011).

Dairy products such as yogurts and cheeses are considered among the most suitable carriers of probiotic strains for human consumption (Lourens-Hattingh and Viljoen, 2001). Because of its dual role as an indigenous human gut microbiota as well as a safe starter culture in food fermentation, L. plantarum exhibits great potential to be applied in dairy systems, including development of various functional dairy products ( $\mathrm{Li}$ et al., 2017). Potential probiotic strains of L. plantarum could be used in starter cultures or used as adjunct bacteria without adverse effects on product quality or sensory properties of fermented milk products (Dan et al., 2019). Many recent studies have reported that consuming L. plantarum-containing fermented milk exerts in vivo functionality to the host (Nasrabadi et al., 2011; Kim et al., 2018).
In our previous study, we screened candidate probiotic strains for anti-inflammatory activity. As a result, 4 candidate probiotic strains (L. plantarum CAU1054, CAU1055, CAU1064, and CAU1106) were selected based on inhibition of NO in LPS-induced RAW264.7 cells, the results of probiotic property assessments, as well as ability to ferment milk, which is a necessary feature for application in dairy systems (Lee et al., 2015). Herein, we investigated and compared the in vitro anti-inflammatory activity of the PGN fraction of novel strains of LAB in murine macrophage RAW264.7 cells and confirmed our findings in a dextran sulfate sodium (DSS)-induced colitis animal model. We also determined the regulatory effect of molecular markers known to mediate inflammatory processes.

\section{MATERIALS AND METHODS}

\section{Reagents}

RAW264.7 cells (murine macrophages) were purchased from Korean Cell Line Bank (KCLB; Seoul, Korea). Dulbecco's modified Eagle's medium and fetal bovine serum were purchased from Gibco (Grand Island, NY). Griess reagent was obtained from SigmaAldrich (St. Louis, MO). Goat anti-rabbit IgG-horseradish peroxidase (HRP), donkey anti-goat IgG-HRP, and goat anti-mouse IgG-HRP antibodies, iNOS, and COX-2 were purchased from Santa Cruz Biotechnology (Santa Cruz, CA), and $\beta$-actin antibody was purchased from Sigma-Aldrich. The ELISA kits for TNF- $\alpha$ and IL-6 were obtained from Thermo Scientific Fermentas (Vilnius, Lithuania) and Raybiotech (Norcross, GA), respectively. Dextran sulfate sodium was from MP Biomedicals (Illkirch, France). Five-week-old male ICR mice were obtained from Young Bio (Seongnam, Korea).

\section{Strains Used for In Vitro and In Vivo Experiments}

Lactobacillus plantarum (CAU1045, CAU1054, CAU1055, CAU1064, and CAU1106), Weissella viridescens CAU1224, Lactobacillus sakei ssp. sakei CAU1273, and Lactobacillus salivarius CAU1301 were obtained from healthy adults (Table 1) and from our laboratory stocks.

For in vitro assays, bacterial cultures were boiled at $95^{\circ} \mathrm{C}$ for $30 \mathrm{~min}$ in $4 \% \mathrm{SDS}$ (Amresco, Solon, $\mathrm{OH}$ ). To extract the PGN fraction from the selected 8 novel strains (Table 1), the insoluble materials were washed 5 times with distilled water, suspended in $10 \mathrm{~mL}$ of 100 $\mathrm{m} M$ Tris-HCl (pH 7.5), and treated with DNase I (5 
$\mu \mathrm{L}, 1 \mathrm{mg} / \mathrm{mL})$, RNase A ( $5 \mu \mathrm{L}, 5 \mathrm{mg} / \mathrm{mL})$, and $\mathrm{MgSO}_{4}$ $(20 \mathrm{mM})$ at $37^{\circ} \mathrm{C}$ for $2 \mathrm{~h}$. Next, trypsin $(10 \mu \mathrm{L}, 10 \mathrm{mg} /$ $\mathrm{mL})$ and $\mathrm{CaCl}_{2}(10 \mathrm{mM})$ were added, and incubation was continued at $37^{\circ} \mathrm{C}$ for $12 \mathrm{~h}$. After centrifugation $(12,000 \times g$ for $15 \mathrm{~min}$ at room temperature), the pellets were boiled in 1\% SDS for $10 \mathrm{~min}$, suspended in $10 \%$ trichloroacetic acid at $4^{\circ} \mathrm{C}$ for $24 \mathrm{~h}$, and washed several times with distilled water until the $\mathrm{pH}$ was neutral. The PGN was stored at $-20^{\circ} \mathrm{C}$ (Wu et al., 2013).

For in vivo assays, Lactobacillus strains were incubated in de Man, Rogosa, and Sharpe broth at $37^{\circ} \mathrm{C}$ and resuspended in PBS at a concentration of $4 \times 10^{10}$ $\mathrm{cfu} / \mathrm{mL}$.

\section{Cell Culture and LAB Treatment}

As described previously (Lee et al., 2015), RAW264.7 cells were incubated in Dulbecco's modified Eagle's medium with $10 \%$ fetal bovine serum and $1 \%$ streptomycin and penicillin at $37^{\circ} \mathrm{C}$ in a humidified $5 \% \mathrm{CO}_{2}$ incubator. The cells were counted using Trypan blue (Amresco) and a hemocytometer. The RAW264.7 cells were plated at a density of $1.5 \times 10^{6}$ cells/well in a $60-\mathrm{mm}$ dish for $24 \mathrm{~h}$; after LPS (100 ng/mL) and PGN $(1,000 \mu \mathrm{g} / \mathrm{mL})$ were added, the incubation was continued for $24 \mathrm{~h}$.

\section{NO Assay}

The production of NO in culture supernatant was measured using Griess reagent, as previously described (Green et al., 1982). About $50 \mu \mathrm{L}$ of the culture supernatant was mixed with equal volumes of Griess reagent for $15 \mathrm{~min}$. Absorbance at $540 \mathrm{~nm}$ was measured using a microplate reader. The concentration of nitrite was determined using a standard curve generated using sodium nitrite (Kim et al., 2007).

\section{Western Blot and ELISA}

Western blotting was conducted as described previously (Bao et al., 2014). Briefly, cell and tissue lysates

Table 1. Lactic acid bacterial strains used in this study

\begin{tabular}{ll}
\hline Strain & Source \\
\hline Lactobacillus plantarum CAU1045 & Lee et al., 2015 \\
Lactobacillus plantarum CAU1054 & Lee et al., 2015 \\
Lactobacillus plantarum CAU1055 & Lee et al., 2015 \\
Lactobacillus plantarum CAU1064 & Lee et al., 2015 \\
Lactobacillus plantarum CAU1106 & Lee et al., 2015 \\
Weissella viridescens CAU1224 & Lee et al., 2015 \\
Lactobacillus sakei ssp. sakei CAU1273 & Lee et al., 2015 \\
Lactobacillus salivarius CAU1301 & Lee et al., 2015 \\
\hline
\end{tabular}

Journal of Dairy Science Vol. 102 No. 8, 2019 were separated on $8 \%$ SDS polyacrylamide gel and transferred onto a polyvinylidene difluoride membrane. The membrane was incubated with primary antibodies of iNOS, COX-2, and $\beta$-actin and then with goat antirabbit IgG-HRP, donkey anti-goat IgG-HRP, and goat anti-mouse IgG-HRP secondary antibodies for $1 \mathrm{~h}$. Enhanced chemiluminescence detection reagent (ATTO, Tokyo, Japan) was used to measure the expression levels of the proteins.

The concentrations of the cytokines TNF- $\alpha$ and IL-6 in the cell culture or tissue lysates were measured using ELISA kits, according to the procedure described by the manufacturers.

\section{Animal Experiment}

Five-week-old male ICR mice were housed in a room with a 12-h light/dark cycle and a temperature of $22 \pm$ $2^{\circ} \mathrm{C}$; they were provided free access to diet and water. The mice were acclimated to the laboratory conditions for $4 \mathrm{~d}$ and used for the animal experiment for $3 \mathrm{wk}$.

The mice were divided into 6 groups of 8 animals each: healthy control group (negative control), DSStreated group (positive control), and Lactobacillustreated groups with DSS. During the animal experiment, a DSS-induced mouse model was used. The IBD control and Lactobacillus groups were fed with $1.5 \%$ DSS instead of drinking water. The healthy control and IBD control were provided $0.2 \mathrm{~mL}$ of PBS, and the Lactobacillus-treated groups received $0.2 \mathrm{~mL}$ of Lactobacillus. Animal experiments were conducted in accordance with the guidelines of the Institutional Animal Care and Use Committee at Chung-Ang University (Approval number: 2017-00103, Seoul, Korea).

\section{Evaluation of IBD and Histological Evaluation}

The BW of the mice was measured at the same time daily to evaluate the degree of IBD. After induction of colitis with DSS, mice were killed under anesthesia. The length of colon from the cecum to the anus was measured. Colon tissues were fixed in $10 \%$ formaldehyde (Duksan, Seoul, Korea) and embedded in paraffin. Sections of the colon tissue were stained with hematoxylin and eosin (H\&E staining), and stained sections were observed using a light microscope $(100 \times)$.

\section{Statistical Analysis}

Data were analyzed using $t$-test by using SPSS statistical software (version 12.0, SPSS Inc./IBM Corp., Chicago, IL). All data are expressed as mean \pm standard error of 3 independent experiments. Differences were considered significant at $P<0.05$. 
(A)

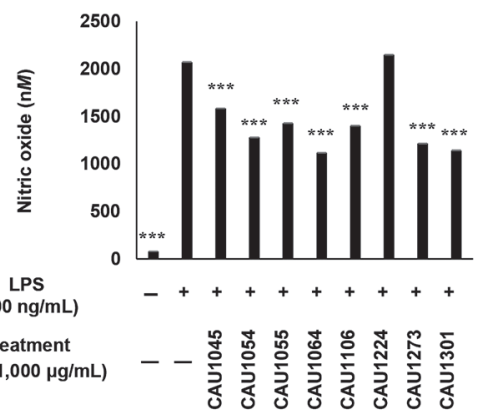

(C)

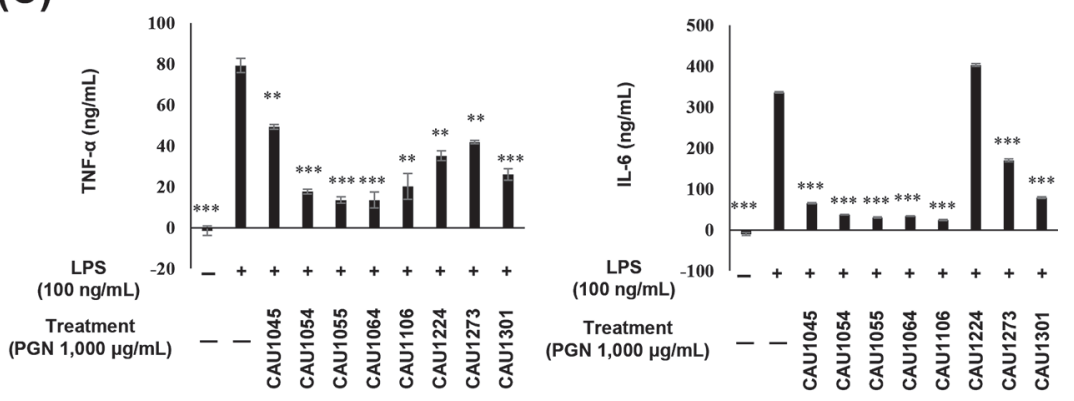

(B)

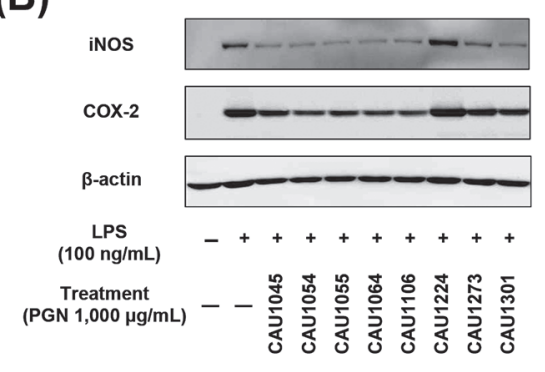

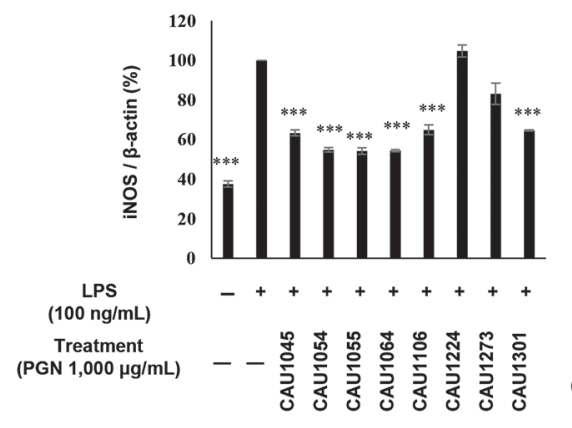

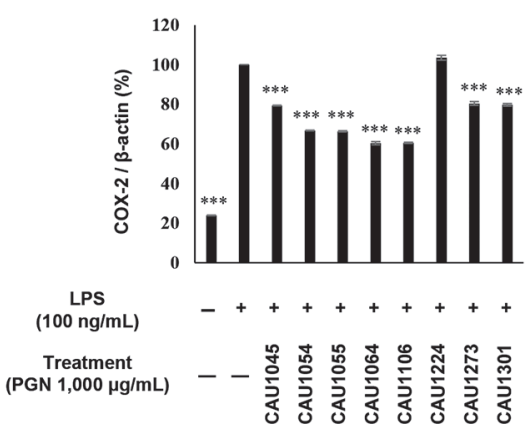

Figure 1. Effects of different strains of probiotics (Lactobacillus plantarum CAU1045, CAU1054, CAU1055, CAU1064, CAU1106; Weissella viridescens CAU1224; Lactobacillus sakei CAU1273; and Lactobacillus salivarius CAU1301) on (A) nitric oxide (NO) synthesis, (B) inducible nitric oxide synthase (iNOS) and cyclooxygenase 2 (COX-2) expression, and (C) cytokines IL-6 and tumor necrosis factor (TNF)- $\alpha$ in LPSinduced RAW264.7 cells. PGN $=$ peptidoglycan extracts. Data are expressed as mean $\pm \mathrm{SE}$ of 3 independent experiments. ${ }^{* *} 0.001<P<0.001$, and ${ }^{* * *} P<0.001$ compared with positive group by $t$-test.

\section{RESULTS AND DISCUSSION}

\section{Effects of Different Strains of Probiotics on NO Synthesis}

Probiotics, including lactobacilli, have been shown to regulate inflammation in a strain-specific manner (Christensen et al., 2002; Foligne et al., 2007). In our previous study, we found that the identified strains had differing efficacies in inhibiting NO synthesis induced by LPS (Lee et al., 2015). Interestingly, Lactobacillus salivarius Ls33 was recently reported to recover 2,4,6-trinitrobenezene sulfonic acid-induced colitis symptoms, where the protective effect was derived from the gram-positive cell wall component PGN (Macho Fernandez et al., 2011), suggesting that muropeptides from strain-specific PGN play key roles in modulating intestinal inflammation (Macho Fernandez et al., 2011). Therefore, we first selected 8 strains (CAU1045, CAU1054, CAU1055, CAU1064, CAU1106, CAU1224, CAU1273, and CAU1301) out of 192 fecal isolates and extracted their PGN fractions to identify the strains with the most significant anti-inflammatory efficacy. After PGN at 500 and $1,000 \mu \mathrm{g} / \mathrm{mL}$ was found to not affect the viability of RAW264.7 cells (data not shown), we investigated the effects of PGN on inhibiting LPS- induced NO production in RAW264.7 cells. All strains except CAU1224 strain ( $W$. viridescens) significantly suppressed NO synthesis compared with the LPS-stimulated NO control (Figure 1A). Of the 7 active strains, the inhibitory activity was as follows: CAU1064 (46\% inhibition), CAU1031 (44.8\%), CAU1273 (41.4\%), CAU1054 (38.2\%), CAU1106 (32.3\%), CAU1055 (31\%), and CAU1045 (23.5\%), indicating that the NO inhibitory effect is strain specific even within the same species (L. plantarum).

\section{Effects of Different Strains of Probiotics on iNOS and COX-2 Expression}

Induction of iNOS by cytokines leads to the production of NO. High concentrations of NO produced by iNOS can become cytotoxic to cells and are involved in inflammatory diseases (Korhonen et al., 2005). To investigate the role of PGN in iNOS expression, we performed Western blot analysis and found that the protein level (expression) of iNOS induced by LPS treatment was suppressed as follows: CAU1055 (45.8\% inhibition), CAU1064 (45.6\%), CAU1054 (45.1\%), CAU1045 (36.6\%), CAU1301 (35.4\%), CAU1106 (35\%), and CAU1273 (16.8\%). Physical and chemical stimuli-induced COX-2 leads to the synthesis of PGN, 
which play critical roles in acute and chronic inflammation and carcinogenesis (Müller-Decker, 2011). Peptidoglycan significantly inhibited COX-2 expression in LPS-induced RAW264.7 cells: CAU1064 (40\% inhibition), CAU1106 (39.5\%), CAU1055 (33.7\%), CAU1054 (33.3\%), CAU1045 (20.6\%), CAU1301 (20.3\%), and CAU1273 (19.6\%; Figure 1B). The strains having NO inhibitory activity (Figure 1A) had similar suppressive activity on LPS-induced iNOS and COX-2 expression in RAW264.7 cells. Consistent with our results, Wu et al. (2013) reported that PGN from Lactobacillus acidophilus showed anti-inflammatory activity by inhibiting expression of iNOS and COX-2.

\section{Effects of Different Strains of Probiotics on IL-6 and TNF- $\alpha$}

Cytokines, mainly produced by different cell types of the immune system, including macrophages and dendritic cells, play critical roles in regulating the initial immune response by a pathogen (Arango Duque and Descoteaux, 2014). Among them, TNF- $\alpha$ is reported to be highly expressed in the colonic tissue and serum of IBD patients and is known to be involved in the regulation of tight junctions and induction of apoptosis in intestinal cells (Ślebioda and Kmiec, 2014). In addition, IL-6 is a proinflammatory cytokine, which was shown to be overexpressed in patients with Crohn's disease (Nishimoto and Kishimoto, 2004). These findings suggest that TNF- $\alpha$ and IL- 6 might be practical molecular targets for treating inflammatory diseases, including IBD. In this study, the expression of LPSinduced TNF- $\alpha$ and IL- 6 was decreased by PGN treatment in the following order for TNF- $\alpha$ : CAU1055 (83\% inhibition), CAU1064 (83\%), CAU1054 (77.7\%), CAU1106 (74.6\%), CAU1301 (67.2\%), CAU1224 (55.7\%), CAU1273 (47.2\%), CAU1045 (37.8\%), and for IL-6: CAU1106 (92.8\% inhibition), CAU1055 (91\%), CAU1064 (90\%), CAU1054 (89\%), CAU1045 (80.5\%), CAU1301 (76.6\%), and CAU1273 (49.8\%; Figure 1C), indicating that PGN of probiotics characterized herein, especially that of CAU1055, CAU1064, and CAU1106, had high efficacy in regulating inflammatory processes in macrophage cells.

\section{Effects of Different Strains of Probiotics on DSS-Induced Colitis Animal Model}

To further investigate in vivo anti-inflammatory activities, we chose 4 strains, CAU1054, CAU1055, CAU1064, and CAU1106, to test a DSS-induced colitis model. As an model to mimic human IBD, DSS is the most commonly used chemical irritant to induce intestinal colitis, resulting in symptoms such as BW loss, bloody diarrhea, colon shortening, and mucosal inflammation, which are similar to those found in humans (Okayasu et al., 1990; Elson et al., 2005). We first tested different concentrations of DSS $(1.5 \%$ and $2.5 \%$ ) to find the optimal concentration of DSS to induce colitis and found that 2.5\% DSS for 3 wk was too toxic in ICR mice. After treatment with $1.5 \%$ DSS, we noted significant decreases in BW (from $32.35 \pm 0.98 \mathrm{~g}$ to $30.53 \pm 0.87 \mathrm{~g}$ ) and colon length (from $10.13 \pm 0.36$ $\mathrm{cm}$ to $6.7 \pm 0.19 \mathrm{~cm}$ ) in ICR mice (Figure 2A). Oral administration of $L$. plantarum CAU1055 significantly increased BW $(32.78 \pm 0.41 \mathrm{~g})$ compared with the DSS-treated group $(30.53 \pm 0.87 \mathrm{~g})$, but the effects of other strains on BW were minimal (Figure 2A). The reduced colon length induced by DSS was most strongly recovered by L. plantarum CAU1055 (from $6.7 \pm 0.19$ $\mathrm{cm}$ to $9.27 \pm 0.08 \mathrm{~cm}$; Figure $2 \mathrm{~B}$ ), indicating that this strain is an important candidate to modulate intestinal colitis induced by DSS.

\section{Effects of L. plantarum CAU1055 on Inflammatory Mediators in DSS-Induced Colitis Model}

To investigate the regulatory mechanisms of L. plantarum strain CAU1055 in inflammatory colon tissues, we performed H\&E staining and protein expression analysis. The colonic tissue in the control group showed normal epithelium and mucosal layer, but extensive disruption of the epithelium (indicated by arrows) and infiltration of inflammatory cells (indicated by stars) were observed in the DSS-treated group (Figure 3A). However, after treatment with L. plantarum strain CAU1055, the mucosal damage was relieved and infiltration of immune cells was rarely observed (Figure 3A), suggesting that L. plantarum strain CAU1055 protects against intestinal inflammatory symptoms induced by DSS.

Several studies on transcriptome analysis have revealed that DSS induces proinflammatory cytokines and their receptors, matrix metalloproteinase family members, cell proliferation-related genes, and well-known mediators such as iNOS and COX-2 (Lee et al., 2009; Cho et al., 2011). The enhanced expression of iNOS and COX-2 by DSS in colon tissues was suppressed by up to 43.4 and $42.7 \%$, respectively, by the administration of the L. plantarum strain CAU1055 (Figure 3B). In addition, the production of proinflammatory cytokines IL-6 and TNF- $\alpha$ in tissue lysates following induction by DSS was decreased from $65.60 \pm 1.96$ to $1.95 \pm 1.48 \mathrm{pg} / 240$ ng of lysate and from $581.5 \pm 28.28$ to $378.17 \pm 18.00$ $\mathrm{pg} / 240 \mathrm{ng}$ of lysate, respectively, after administration of L. plantarum CAU1055 (Figure 3C). Considering 
the results of in vitro and in vivo studies, we propose that L. plantarum strain CAU1055 ameliorates colonic inflammation by modulating iNOS, COX-2, IL-6, and TNF- $\alpha$. In agreement with the findings of the present

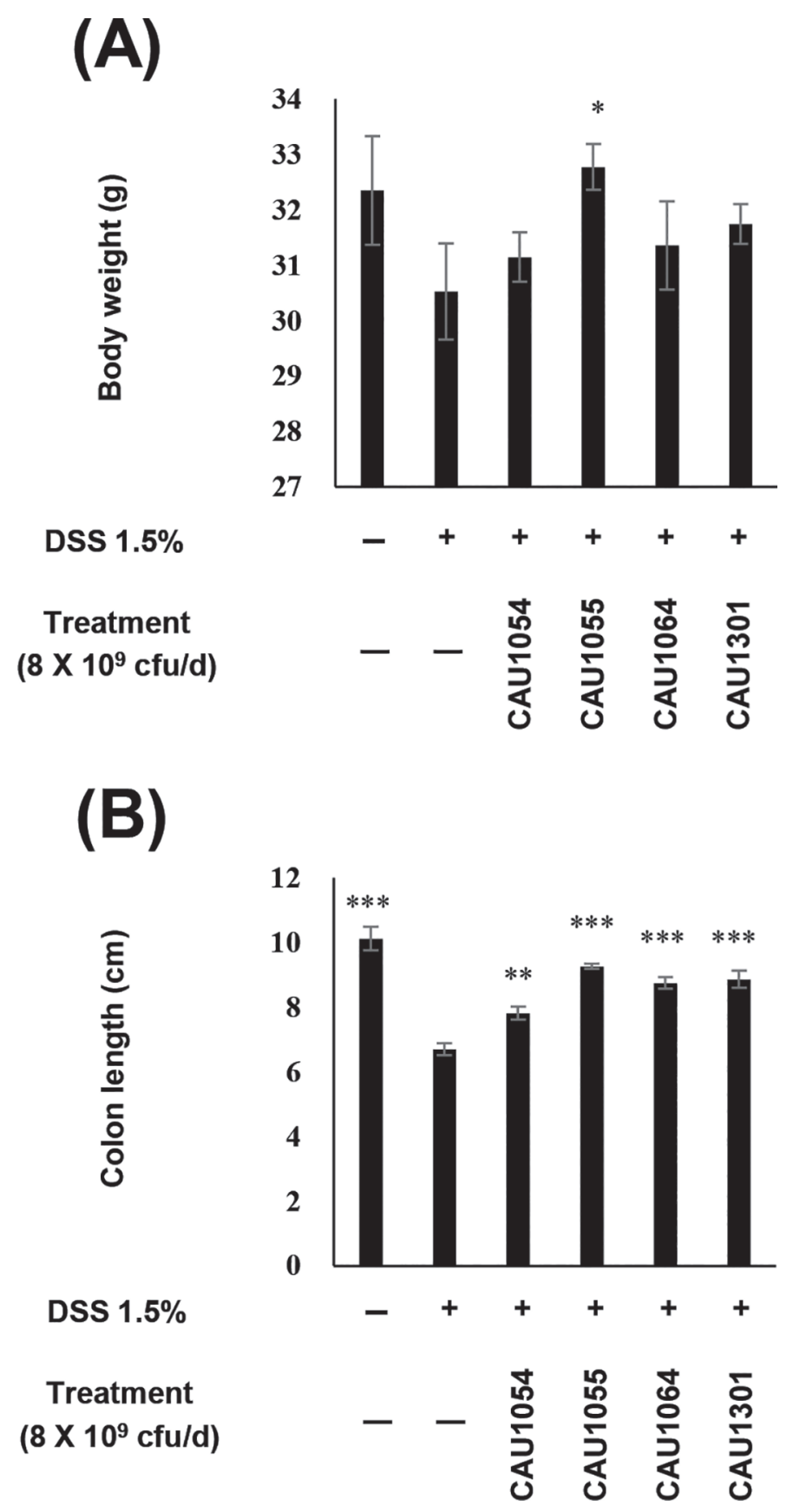

Figure 2. Effects of different strains of probiotics (Lactobacillus plantarum CAU1054, CAU1055, CAU1064; and Lactobacillus salivarius CAU1301) on (A) BW and (B) colon length in a dextran sulfate sodium (DSS)-induced colitis mouse model at d 21. Data are expressed as mean $\pm \mathrm{SE}$ of 3 independent experiments. ${ }^{*} 0.01<P<$ $0.05,{ }^{* *} 0.001<P<0.001$, and ${ }^{* * *} P<0.001$ compared with positive group by $t$-test. study, several studies have indicated that strain-specific probiotics, including Lactobacillus fermentum BR11 and L. rhamnosus GG (Geier et al., 2007), L. plantarum K68 (Liu et al., 2011), and L. plantarum Lp91 (Duary et al., 2012), show significant immunomodulatory effects in DSS-induced animal models, indicating that the novel strain of $L$. plantarum isolated from human feces in the current study may also play a crucial role in modulating inflammatory progression in the colon.

The molecular mechanism of immune modulation by microorganisms is not yet clearly elucidated (Weiss et al., 2010). Peptidoglycan, the main component of grampositive bacteria cell wall, consists of alternating residues of $\beta$-(1,4)-linked $N$-acetylglucosamine (GlcNAc) and $\mathrm{N}$-acetylmuramic acid (MurNAc) and L- and Damino acids. The short peptide chain is cross-linked to the peptide chain of another strand and attached to MurNAc. Generally, the peptide chain is L-Ala- $\gamma$ D-Glu-L-Lys-D-Ala, but this varies depending on microbial species (Chaput and Boneca, 2007). Immunity is controlled by the third amino acid of the peptide chain and the variation in cross-linking to the peptide. Thus, the signaling pathway induced by the interaction of microbe-associated molecular patterns and pattern recognition receptors (such as TLR2/TLR6; Ren et al., 2016) can be controlled variably depending on the microorganism. Diseases such as IBD and allergic reactions can be treated and prevented by this interaction.

\section{CONCLUSIONS}

Among the 8 identified strains of 192 fecal isolates, we found that PGN from L. plantarum CAU1054, CAU1055, CAU1064, and CAU1301 had a significant inhibitory effect on LPS-induced inflammation by regulating iNOS, COX-2, IL-6, and TNF- $\alpha$ in RAW264.7 murine macrophage cells. In the DSS-induced inflammatory animal model, we confirmed that L. plantarum strain CAU1055 ameliorated intestinal inflammatory symptoms such as BW loss and colon shortening by modulating the same inflammatory markers in colon tissue. These results indicate that L. plantarum strain CAU1055 can act as a potent and safe probiotic with beneficial effects on inflammatory diseases in the colon, although further studies are necessary to clarify the exact mechanisms of interaction between PGN of $L$. plantarum CAU1055 and upstream cellular signaling mediators. Based on the functional properties shown in this study as well as the milk-fermenting characteristic reported in our previous study (Lee al., 2015), we suggest that L. plantarum CAU1055 is a potential probiotic candidate for application in the development of functional dairy products. 
(A)
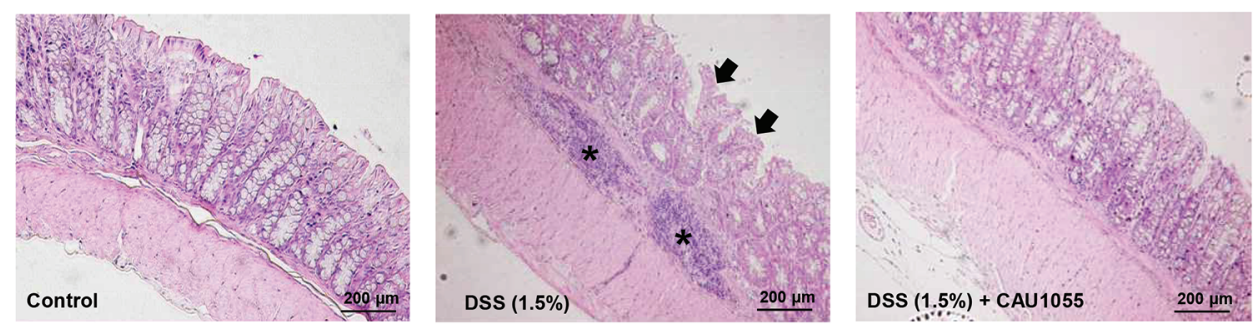

(B)

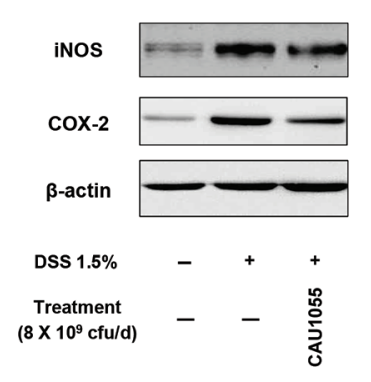

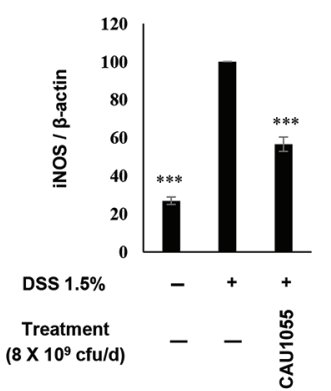

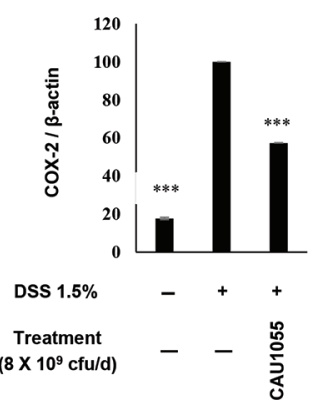

(C)

Figure 3. Effects of Lactobacillus plantarum strain CAU1055 on (A) colonic histology, (B) relative expression of inducible nitric oxide synthase (iNOS) and cyclooxygenase 2 (COX-2), and (C) production of cytokines IL-6 and tumor necrosis factor (TNF)- $\alpha$ in a dextran sulfate sodium (DSS)-induced colitis animal model. In panel A, extensive disruption of the epithelium (arrows) and infiltration of inflammatory cells (stars) were observed in the DSS-treated group. After treatment with L. plantarum strain CAU1055, the mucosal damage was relieved and infiltration of immune cells was rarely observed. Data are expressed as mean $\pm \mathrm{SE}$ of 3 independent experiments. ${ }^{* *} 0.001<P<0.001$, and ${ }^{* * *} P$ $<0.001$ compared with positive group by $t$-test.

\section{ACKNOWLEDGMENTS}

This work was supported by the Korea Institute of Planning and Evaluation for Technology in Food, Agriculture, Forestry and Fisheries (IPET) through the Agri-Bio Industry Technology Development Program, funded by the Ministry of Agriculture, Food and Rural Affairs (MAFRA) (318091-03-1-HD030). This research was also supported by the Chung-Ang University Graduate Research Scholarship in 2017.

\section{REFERENCES}

Arango Duque, G., and A. Descoteaux. 2014. Macrophage cytokines: Involvement in immunity and infectious diseases. Front. Immunol. $5: 491$.

Atreya, R., J. Mudter, S. Finotto, J. Mullberg, T. Jostock, S. Wirtz, M. Schütz, B. Bartsch, M. Holtmann, C. Becker, D. Strand, J. Czaja, J. F. Schlaak, H. A. Lehr, F. Autschbach, G. Schürmann, N. Nishimoto, K. Yoshizaki, H. Ito, T. Kishimoto, P. R. Galle, S. Rose-John, and M. F. Neurath. 2000. Blockade of interleukin 6 trans signaling suppresses T-cell resistance against apoptosis in chronic intestinal inflammation: Evidence in Crohn disease and experimental colitis in vivo. Nat. Med. 6:583-588.

Bao, C., H. Namgung, J. Lee, H. C. Park, J. Ko, and H. Moon. 2014. Daidzein suppresses tumor necrosis factor-alpha induced migration and invasion by inhibiting hedgehog/Gli1 signaling in human breast cancer cells. J. Agric. Food Chem. 62:3759-3767.
Campana, R., R. van Hemert, and W. Baffone. 2017. Strain-specific probiotic properties of lactic acid bacteria and their interference with human intestinal pathogens invasion. Gut Pathog. 9:12.

Chaput, C., and I. G. Boneca. 2007. Peptidoglycan detection by mammals and flies. Microbes Infect. 9:637-647.

Chin, A. C., and C. A. Parkos. 2006. Neutrophil transepithelial migration and epithelial barrier function in IBD: potential targets for inhibiting neutrophil trafficking. Ann. N. Y. Acad. Sci. 1072:276287.

Cho, J. Y., J. K. Hwang, and H. S. Chun. 2011. Xanthorrhizol attenuates dextran sulfate sodium-induced colitis via the modulation of the expression of inflammatory genes in mice. Life Sci. 88:864-870.

Christensen, H. R., H. Frokiaer, and J. J. Pestka. 2002. Lactobacilli differentially modulate expression of cytokines and maturation surface markers in murine dendritic cells. J. Immunol. 168:171-178.

Cui, Y., H. Wei, F. Lu, X. Liu, D. Liu, L. Gu, and C. Ouyang. 2016. Different effects of three selected Lactobacillus strains in dextran sulfate sodium-induced colitis in BALB/c mice. PLoS One 11:e0148241.

Dan, T., H. Chen, T. Li, J. Tian, W. Ren, H. Zhang, and T. Sun. 2019. Influence of Lactobacillus plantarum P-8 on fermented milk flavor and storage stability. Front. Microbiol. 9:3133.

Dinarello, C. A. 2006. The paradox of pro-inflammatory cytokines in cancer. Cancer Metastasis Rev. 25:307-313.

Duary, R. K., M. A. Bhausaheb, V. K. Batish, and S. Grover. 2012. Anti-inflammatory and immunomodulatory efficacy of indigenous probiotic Lactobacillus plantarum Lp91 in colitis mouse model. Mol. Biol. Rep. 39:4765-4775.

Ebrahimi, M. T., and P. Jafarei. 2011. Lactobacillus acidophilus cell structure and application. Afr. J. Microbiol. Res. 5:4033-4042.

Elson, C. O., Y. Cong, V. J. McCracken, R. A. Dimmitt, R. G. Lorenz, and C. T. Weaver. 2005. Experimental models of inflammatory 
bowel disease reveal innate, adaptive, and regulatory mechanisms of host dialogue with the microbiota. Immunol. Rev. 206:260-276.

Foligne, B., S. Nutten, C. Grangette, V. Dennin, D. Goudercourt, S. Poiret, and J. Dewulf., D. Brassart, A. Mercenier, and B. Pot 2007. Correlation between in vitro and in vivo immunomodulatory properties of lactic acid bacteria. World J. Gastroenterol. 13:236-243.

Fong, F. L. Y., P. Kirjavainen, V. H. Y. Wong, and H. El-Nezami. 2015. Immunomodulatory effects of Lactobacillus rhamnosus GG on dendritic cells, macrophages and monocytes from healthy donors. J. Funct. Foods 13:71-79.

Geier, M. S., R. N. Butler, P. M. Giffard, and G. S. Howarth. 2007. Lactobacillus fermentum BR11, a potential new probiotic, alleviates symptoms of colitis induced by dextran sulfate sodium (DSS) in rats. Int. J. Food Microbiol. 114:267-274.

Green, L. C., D. A. Wanger, J. Glogowski, J. Skipper, J. S. Wishnok, and S. R. Tannenbaum. 1982. Analysis of nitrate, nitrite, and $\left[{ }^{15} \mathrm{~N}\right]$ nitrate in biological fluids. Anal. Biochem. 126:131-138.

Hijova, E., and A. Soltesova. 2013. Effects of probiotics and prebiotics in ulcerative colitis. Bratisl. Lek Listy 114:540-543.

Kim, D. H., W. C. Chung, S. H. Chun, J. H. Han, M. J. Song, and K. W. Lee. 2018. Enhancing the natural killer cell activity and anti-influenza effect of heat-treated Lactobacillus plantarum $\mathrm{nF1-}$ fortified yogurt in mice. J. Dairy Sci. 101:10675-10684.

Kim, D. W., S. B. Cho, C. H. Yun, H. Y. Jeong, W. T. Chung, and C. W. Choi. 2007. Induction of cytokines and nitric oxide in murine macrophages stimulated with enzymatically digested Lactobacillus strains. J. Microbiol. 45:373-378.

Kim, J.-E., C. S. Chae, G.-C. Kim, W. Hwang, J.-S. Hwang, and S.M. Hwang. 2015. Lactobacillus helveticus suppresses experimental rheumatoid arthritis by reducing inflammatory $\mathrm{T}$ cell responses. J. Funct. Foods 13:350-362.

Korhonen, R., A. Lahti, H. Kankaanrata, and E. Moilanen. 2005. Nitric oxide production and signaling in inflammation. Curr. Drug Targets Inflamm. Allergy 4:471-479.

Lee, H., Y. T. Ahn, J. H. Lee, C. S. Huh, and D. H. Kim. 2009. Evaluation of anti-colitic effect of lactic acid bacteria in mice by cDNA microarray analysis. Inflammation 32:379-386.

Lee, H. K., S. H. Choi, C. R. Lee, S. H. Lee, M. R. Park, Y. Kim, M. K. Lee, and G.-B. Kim. 2015. Screening and characterization of lactic acid bacteria strains with anti-inflammatory activities through in vitro and Caenorhabditis elegans model testing. Korean J. Food Sci. Anim. Resour. 35:91-100.

Li, C., J. Song, L.-Y. Kwok, J. Wang, Y. Dong, H. Yu, Q. Hou, H. Zhang, and Y. Chen. 2017. Influence of Lactobacillus plantarum on yogurt fermentation properties and subsequent changes during postfermentation storage. J. Dairy Sci. 100:2512-2525.
Liu, Y. W., Y. W. Su, W. K. Ong, T. H. Cheng, and Y. C. Tsai. 2011. Oral administration of Lactobacillus plantarum K68 ameliorates DSS-induced ulcerative colitis in $\mathrm{BALB} / \mathrm{c}$ mice via the anti-inflammatory and immunomodulatory activities. Int. Immunopharmacol. 11:2159-2166.

Lourens-Hattingh, A., and B. C. Viljoen. 2001. Review: Yoghurt as probiotic carrier in food. Int. Dairy J. 11:1-17.

Macho Fernandez, E., V. Valenti, C. Rockel, C. Hermann, B. Pot, I. G. Boneca, and C. Grangette. 2011. Anti-inflammatory capacity of selected lactobacilli in experimental colitis is driven by NOD2mediated recognition of a specific peptidoglycan-derived muropeptide. Gut 60:1050-1059.

Medzhitov, R. 2008. Origin and physiological roles of inflammation. Nature 454:428-435.

Müller-Decker, K. 2011. Cyclooxygenase-dependent signaling is causally linked to non-melanoma skin carcinogenesis: pharmacological, genetic, and clinical evidence. Cancer Metastasis Rev. 30:343-361.

Nasrabadi, M. H., H. Aboutalebi, M. T. Ebrahimi, and F. Zahedi. 2011. The healing effect of Lactobacillus plantarum isolated from Iranian traditional cheese on gastric ulcer in rats. Afr. J. Pharm. Pharmacol. 5:1446-1451.

Nishimoto, N., and T. Kishimoto. 2004. Inhibition of IL-6 for the treatment of inflammatory diseases. Curr. Opin. Pharmacol. 4:386-391.

Okayasu, I., S. Hatakeyama, M. Yamada, T. Ohkusa, Y. Inagaki, and R. Nakaya. 1990. A novel method in the induction of reliable experimental acute and chronic ulcerative colitis in mice. Gastroenterology 98:694-702.

Ren, C., Q. Zhang, B. J. de Haan, H. Zhang, M. M. Faas, and P. de Vos. 2016. Identification of TLR2/TLR6 signalling lactic acid bacteria for supporting immune regulation. Sci. Rep. 6:34561.

Ślebioda, T. J., and Z. Kmiec. 2014. Tumour necrosis factor superfamily members in the pathogenesis of inflammatory bowel disease. Mediators Inflamm. 2014:325129.

Wang, G., Y. Liu, Z. Lu, Y. Yang, Y. Xia, P. F.-H. Lai, and L. Ai. 2019. The ameliorative effect of a Lactobacillus strain with good adhesion ability against dextran sulfate sodium-induced murine colitis. Food Funct. 10:397.

Weiss, G., S. Rasmussen, L. H. Zeuthen, B. N. Nielsen, H. Jarmer, L. Jespersen, and H. Frokiaer. 2010. Lactobacillus acidophilus induces virus immune defense genes in murine dendritic cells by a Toll-like receptor-2-dependent mechanism. Immunology 131:268-281.

Wu, Z., D.-D. Pan, Y. Guo, and X. Zeng. 2013. Structure and anti-inflammatory capacity of peptidoglycan from Lactobacillus acidophilus in RAW-264.7 cells. Carbohydr. Polym. 96:466-473. 